\title{
Discursos Sociales Disonantes y la Reformulación Identitaria en un Centro Integral del Adulto Mayor de Quilicura
}

\author{
doi: 10.33264/rpa.202101-03 \\ Jesús Aparicio de Soto \\ Susana Cornejo Farías \\ Francisca Lain Soteras \\ Paula Mallegas Roblin \\ Prof. Guía: Paulina Soto Vásquez \\ Escuela de Psicología UNIACC \\ Facultad de Humanidades y Ciencias Sociales UNIACC
}

\section{Resumen}

Este estudio buscó comprender los significados atribuidos a los discursos sociales respecto del envejecimiento en un grupo de adultos y adultas mayores del Centro Integral de Quilicura, y la relevancia de estos en el plano emocional y social. Se utilizó la entrevista semiestructurada como instrumento, y un análisis basado en la teoría fundamentada. Los resultados mostraron dos elementos muy relevantes del discurso social; la injusticia social y el miedo a la dependencia, los que movilizan significados disonantes entre lo que es promovido por la sociedad y las oportunidades reales que se imparten en esta, siendo esto trascendental en la reformulación identitaria y la afectividad de los adultos mayores.

Palabras clave: adulto mayor, discurso social, identidad, significado.

\begin{abstract}
Using semi-structured interviews and an analytic approach based on the grounded theory, this research took on constructing an understanding of the socio-emotional relevance, and the meaning that a group of elder participants of CIAM «Lucía Salas Romo» of Quilicura (Santiago, Chile) ascribe to social discourses regarding aging. Findings showed that, mobilizing the semantic dissonance between what society promotes and the effective opportunities it allows for the elderly, there are two crucial components of the social discourse: social injustice and fear of dependency. In turn, these play a central role in elder affectivity and the reformulation of identity during this developmental stage for this group.
\end{abstract}

Keywords: elderly, social discourse, identity, meaning. 


\section{Los desafíos que trae una sociedad que envejece}

El desarrollo humano está determinado por la continua convergencia de cambios en las formas de ser, estar y relacionarse, personales y en contexto. Freud, Erikson, Piaget y Kohlberg, dividen el envejecimiento en etapas, siempre dentro del entorno social.

“«Hacerse grande», ha sido casi siempre, a lo largo de la historia, algo deseable, (...) «hacerse viejo» ha tenido connotaciones negativas" (Moñivas, 1998, p. 17). Durante la adultez mayor, simplificado cómo AM, la flexibilidad para poder participar y reformularse prueba ser esencial.

Cómo política e ideal a perseguir, el "envejecimiento activo ayuda a cambiar la percepción negativa y fatalista de la vejez (...) [ya que] urge cambiar la visión" (Rojas, 2017, pp. 8-9). Toman así valor los discursos sociales (DDSS), por su significación biopsicosocial. El discurso social, abreviado DS, está compuesto de "prácticas lingüísticas que mantienen y promueven ciertas relaciones" (Iñiguez \& Antaki, 1994, p. 63): habilita e incide en el pensamiento.

De acuerdo al INE, en Chile, en 1992, la población menor de quince años era cinco veces mayor al número de adultos mayores (en adelante $A A M M$ ), lo que se espera en 2031 alcance a un AM por cada menor (Patillo, 2018). Llamaremos adulto mayor, que también referiremos $\mathrm{AM}$, a quién tenga sobre sesenta y cinco años de edad. Ante la proyección, se vuelve un desafío generar políticas públicas atingentes y una mirada positiva, no deficitaria del envejecimiento.

Arnold, Thumala, Urquiza y Ojeda (2007) develaron que mayormente los jóvenes ven la vejez como una desgracia, "una lucha por aferrarse a las cualidades propias de una juventud y adultez idealizada” (p. 89). Esto propende a la frustración de quienes se acercan a la AM. Además, Herrera et al. (2017) exponen que los AAMM tienen una percepción de un trato injusto por el hecho de ser mayores.

Como motivación entonces, se presentan: el aumento de la población AM, la percepción de trato injusto, los DDSS, ausencia de roles participativos, mirada deficitaria del envejecimiento, exclusión productiva, jubilación, incertidumbre, mitos y prejuicios respecto de la AM.

\section{El recorrido de la identidad social durante la adultez mayor}

El envejecimiento implica cambios. Se comprenderá este proceso integrada y holísticamente: cómo una permanente interrelación entre el ser y su contexto, esencial en la identidad.

El desarrollo es un proceso continuo, dinámico, situado, que requiere de gran 
flexibilidad (lacub, 2014). Una diversidad de trayectorias socio-identitarias divergentes pues "las incongruencias entre las normas sociales y el ciclo de vida fluido representan nuevas libertades para unos, [pero] para otros nuevas incertidumbres" (Neugarten, 1999, p. 82).

Aquí Erikson (2000, en lacub, 2014), destaca una serie de procesos que “desencadenarán sintonía o distonía en un equilibrio siempre dinámico. (...) [poniendo en juego] deseos y temores, seguridades e inseguridades, necesidades y carencias" (p.198). Estas elaboraciones se van articulando, resignificando y actualizando. Para Erikson (1998, en Bordignon, 2005), el último estadio presenta el desafío de la integridad; la aceptación del sí mismo; la historia personal; la integración emocional de la confianza y la autonomía, y de las contribuciones significativas a la humanidad, vislumbrado de qué manera se va a trascender.

Para Izquierdo (2006), la psicología del desarrollo explica cambios del "pensamiento, vivencias y conductas” (p.602), esperándose en la AM la jubilación, pérdidas y/o enfermedades. Al extenderse la expectativa de vida, se requerirán "rerritualizaciones vitales, que (...) [generen] algún sentimiento finito de síntesis” (Erikson, 1985, p. 79).

El envejecimiento conlleva transformaciones biopsicosociales que desafían la identidad. Los AAMM deben adaptarse permanentemente para mantener un sí mismo coherente (lacub, 2014). Para Piaget (1983, citado en lacub 2014) debe armonizarse la asimilación y la acomodación, modificándose esquemas mentales buscando la integridad. Cuando las personas comienzan a sentirse viejas, se debe a cambios identitariamente contradictorios. Estos pueden estar agrupados en "representaciones prejuiciosas que [los] significan negativamente" (lacub, 2014, p. 144) pudiendo así resonar con los DDSS.

Los estereotipos confunden los cambios etarios esperables, con los patológicos (lacub, 2014). Muchos asimilan la AM como enfermedad, no un tiempo de vivir con las mismas energías. Ver a los AAMM como dependientes aun si no lo son, genera apreciaciones que pueden perturbar la percepción personal respecto de sus propios vínculos. Se debe buscar un significado de vejez que abarque el bienestar biopsicosocial y no sólo las "pérdidas», una reconstrucción identitaria basada en la experiencia biográfica, generando acumulación y desarrollo de cambios, creatividad y calidad de vida a través de las relaciones.

\section{La integración ante un discurso deficitario del envejecimiento}

Los DDSS están formados por expectativas ante situaciones específicas y la cultura popular (Charmaz, 2006, p. 120). Sostienen pensamientos (Young \& Collin, 2004, p. 379) y "expresan compromisos comunicacionales hacia la práctica" (Sandoval, 1997, 
p. 4). Para Mead (2008), "una dialéctica continua de conversión de la imagen del mundo" (p. 56). El DS juega un papel "tanto para sustentar pautas establecidas de conducta colectiva como para propiciar su transformación" (Blumer, 1982, pp. 4950).

Al "interior de un discurso, la separación entre lo verdadero y lo falso no es ni arbitraria, ni modificable (...) Pero si uno se sitúa en otra escala, (...) ve dibujarse algo así como un sistema de exclusión” (Foucault, 2005, p. 15). En efecto, Margaret Mead (2006) señala que "nuestra propia cultura ha confiado (...) en muchas distinciones artificiales, la más notable de las cuales es la del sexo (...) Si queremos una cultura más rica en valores contrastantes, debemos reconocer toda la escala de las potencialidades" (p. 221).

"La «radiografía» cultural que nos presenta la Encuesta Mundial de Valores y el Latinobarómetro del Chile actual nos describe un país que se caracteriza por una desconfianza social" (Benito, 2013, pp. 113-114), más "el control de las condiciones de producción de los DDSS (... ) [determina] la posibilidad de participar" (Sandoval, 1997, p. 4). Pero ¿cómo el AM da sentido y significa estos DDSS? ¿cómo afecta ello su participación?

“Para Piaget (1983) debería haber un equilibrio (...) [dónde] la asimilación debe dar gradualmente lugar al proceso de acomodación (...) [manteniendo] un sí mismo coherente" pero los cambios al envejecer suelen entenderse de forma tendenciosa (lacub, 2014, p. 144). Si el DS sostiene interpretaciones negativas y los AAMM las han asimilado: se estructuran esquemas cognitivos que dificultan la construcción de la identidad y la percepción de autoeficacia. Papalia, Feldman y Martorell (2012), destacan que el AM recurre más a la memoria de largo plazo y probablemente a costumbres y recuerdos para pensar. La exposición a DDSS negativos podría condicionar a no disfrutar de envejecer, a lo que lacub (2014) contrapone que la vida se incrementa en 7,5 años si es que hay una percepción positiva de la vejez.

Siguiendo a Bandura (1977, citado en lacub 2014), los AAMM, al percibirse dentro de un grupo estereotipado negativamente, van "limitando progresivamente la autoeficacia” (p. 127) emergiendo la evitación. Aho (2018) la caracteriza como una enajenación que extravía las decisiones y proyectos debido a las expectativas negativas (pp. 7-8). Papalia et al. (2012) agregan que la negatividad dificulta además el procesamiento cognitivo.

En Chile hay "convergencia entre los distintos discursos, "cuando se transforma lo social y lo humano en capital»" (Sandoval, 2009, pp. 47-48). Si, Ortega (2018) presenta la vejez entendida como "carga», esto pareciera afectar directamente a la población AM ya que, siguiendo a Gergen (2007), “el modernista (...) desdeñará a los 
ancianos y a los enfermizos, pues [desde su perspectiva] no cumplen ninguna función útil” (p. 335).

\section{La posibilidad de depender y sus alcances psicoafectivos}

El DS podría estar condicionando a los AAMM a no disfrutar. Predomina un sistema que extrae al $A M$ del circuito productivo y tiende a mirarlos deficitariamente provocando la "falta de inserción social, la carencia de roles específicos y el progresivo aislamiento" (lacub, 2014, p. 188). Según Herrera et al. (2017), dentro de las mayores preocupaciones del AM está depender. Pero significar la pérdida de funcionalidad cómo padecimiento tiende a relacionarse con dependencia (Thumala, 2011) generando miedos, y ansiedades.

Las emociones adquieren un significado relacional que depende de cómo se le da sentido al actuar del otro (Gergen, 1996; Lazarus, 2000). El correlato discursivo explica cómo se estructura la emoción. Según Bandura (1998), la activación afectiva, significará distintamente la ansiedad, rabia o tristeza, si se interpretan cómo amenaza, insulto o pérdida (p. 78).

Si Madariaga et al. (2008) establecen que la inteligencia, la capacidad de aprendizaje, la experiencia, la personalidad y los sentimientos son funciones que no se pierden con la edad, más, se enriquecen en un ambiente propicio. Es necesario alcanzar una visión integrativa, pero el asociar la vejez a un estado de decadencia aún persiste en la sociedad contemporánea.

\section{Problematización: objetivos y pregunta de investigación}

No hay mayor noción respecto de cómo son comprendidos los DDSS referentes al envejecimiento y sus significados por los AAMM, o de su relevancia. Sin embargo, se hace evidente que "promover la interacción social en este periodo es fundamental (... ) [pues] urge cambiar la visión de vejez" (Rojas, 2017, pp. 8-9). Nos preguntamos ¿Cómo son los significados atribuidos a los discursos sociales sobre el envejecimiento en un grupo de adultos y adultas mayores del CIAM de Quilicura?

Luego, el objetivo general es comprender los significados atribuidos a los discursos sociales sobre el envejecimiento en un grupo de adultos y adultas mayores del CIAM de Quilicura. Específicamente: (i.) describir el o los DDSS que los y las AAMM del CIAM de Quilicura conocen sobre el envejecimiento y las creencias asociadas a estos discursos; (ii.) comprender la relevancia emocional que tienen los DDSS sobre el envejecimiento en la descripción que hacen de sí mismos los y las AAMM del CIAM de Quilicura; (iii.) comprender la relevancia que tiene en el ámbito psicosocial de los y las AAMM del CIAM de Quilicura, la significación que hacen de los DDSS del 
envejecimiento, y (iv.) explorar las expectativas que tienen los y las AAMM del CIAM de Quilicura respecto del envejecimiento en Chile.

\section{Tipificación: paradigma y enfoque del estudio}

Se adopta aquí un enfoque cualitativo: indagar las cualidades subyacentes de un fenómeno como un todo integrado (Martínez, 2006). El paradigma epistemológico es constructivista, dirigiéndose a la percepción del AM y a "explicar la formación del conocimiento situándose en el interior del sujeto" (Delval, 1997, p. 355): la significación del DS.

Por medio de la entrevista semiestructurada, se describen propiedades personales (Dankhe 1986, citado en Hernández et al. 2014). "La realidad» "se define a través de las interpretaciones de los participantes en la investigación” (Hernández et al. 2014, p. 60) eventualmente convergiendo hacia propuesta paradigmática. Los participantes son mucho más que «unidades de estudio» aisladas y estáticas: el investigador va construyendo sabiendo que forma parte del fenómeno (Hernández et al. 2014).

El diseño es de tipo no experimental, transversal: la recolección de datos contempla un tiempo único (Hernández et al. 2014). El alcance es exploratorio, caracterizando un fenómeno no ahondado previamente. Es también descriptivo puesto que busca identificar, describir e interpretar los diversos significados que atribuyen los AAMM al DS (Katayama, 2014).

\section{Selección: criterios para seleccionar a los participantes}

Participaron seis personas, cinco mujeres y un hombre, entre 65 y 77 años. Estos debieron cumplir con ser AAMM de 65 años o más, contar con buen estado de salud, considerando el contexto sanitario, tener más de diez años de residencia en Chile y haber accedido voluntariamente a participar. Se requirió incluir tanto a hombres cómo a mujeres, únicamente a personas autovalentes con capacidad para realizar AVD y que representaran diferentes niveles socioeconómicos y culturales.

Se excluyeron deliberadamente personas con discapacidad vocal, auditiva u otra que impidiera desarrollar las entrevistas en la modalidad acordada. También casos en que se impedía la comprensión de contenidos cómo trastornos mentales, demencia, esquizofrenia, trastornos del espectro autista u otros del desarrollo; evidente déficit o deterioro cognitivo que pudiera interferir en la entrega comprensible de las respuestas, o COVID-19.

\section{Validación: elaboración del instrumento de recolección}

Se realizaron entrevistas semiestructuradas, permitiendo acceder a temas relevantes 
para el participante y no a los que la teoría previera. Un guion en consistencia con los objetivos fue validado por una comisión de tres expertos. Sus sugerencias fueron incorporadas.

Respecto de esta forma de entrevistar, Valles (2009) plantea que la "clave (...) está en la incitación de relatos de experiencia vivida durante la entrevista” (p. 98). Así, el instrumento fue reevaluado tras cada entrevista, elaborando ajustes para acceder a los temas relevantes, en concordancia con el muestro teórico de la teoría fundamentada, y a los objetivos.

\section{Recolección: descripción del procedimiento}

Para Noreña et al. (2012), los procedimientos deben documentarse para que eventualmente "otros investigadores puedan reproducir fielmente nuestros pasos" (p. 76). En este caso:

i) La coordinadora del CIAM sondea el interés de AAMM del CIAM en participar y proporciona la lista de interesados. Se les hace llegar vía e-mail y WhatsApp un consentimiento informado explicitando además que las entrevistas son telefónicas.

ii) Cada investigador contacta telefónicamente a un entrevistado por vez, agendando día y hora. Se informa que se grabará, mediante la aplicación «Call Recorder».

iii) Así, primero, se realizan y transcriben dos entrevistas avanzando con una comparación constante de los datos, codificando y analizando. A continuación, luego de tres entrevistas más, se empiezan a reiterar las ideas principales. Finalmente, luego de una sexta entrevista, ya analizada, se produce «saturación teórica», terminándose de definir la muestra (San Martin Cantero, 2014, citado en Bonilla y López, 2016).

\section{Consideraciones de acceso, limitantes y el contexto sanitario}

Es importante dar cuenta del contexto de pandemia de coronavirus, durante la cual se realizaron las entrevistas. La OMS señala que existe poca información sobre la historia natural de la enfermedad, epidemiología e impacto clínico. El COVID-19, comenzó rápidamente a propagarse desde China, posteriormente aumentaron los casos constituyéndose una emergencia internacional (OMS, 2020).

Las medidas del gobierno para los centros del AM apuntan al cierre temporal mientras se mantenga el estado de emergencia. El CIAM de Quilicura orienta sus acciones en conjunto con equipos de salud para ayudar a reducir contagios.

Dado que el estudio se desarrolla bajo estas circunstancias, las entrevistas se realizaron telefónicamente, constituyéndose en una limitante de acceso por los impases técnicos al cortarse la llamada en algunas de las entrevistas. Esta misma 
situación genera que no se hayan podido pesquisar algunos componentes de la comunicación no verbal de los participantes.

\section{Consideraciones éticas respecto a la confidencialidad y los registros}

Los criterios éticos se sustentaron en lo propuesto por Noreña et al. (2012): primero, respecto del (i) consentimiento informado, los participantes de la investigación son informados del propósito y motivación. El carácter de (ii) confidencialidad, se asegura mediante la reserva de la identidad, grabaciones, registros y datos personales. El (iii) manejo de riesgos sigue "los principios de no maleficencia y beneficencia establecidos para hacer investigación con seres humanos" (p. 272), y el criterio de observación participante. Noreña et al. (2012) indican que "la incursión del investigador en el campo exige una responsabilidad ética por los efectos y consecuencias" (p. 272). Los participantes, pudieron resolver no continuar o retirarse sin perjuicio ni explicación, y toda duda respecto de la investigación era respondida y aclarada.

Paulina Soto Vásquez, Psicóloga especialista en este tipo de investigaciones, supervisó el estudio. Se tuvo en cuenta en todo momento el código de ética establecido por el Colegio de Psicólogos de Chile (1999), especialmente el "respeto por los derechos y la dignidad" (p. 7).

\section{Consideraciones analíticas durante la codificación de los datos}

Se aplicó Teoría Fundamentada para recolectar, codificar y analizar buscando la comprensión (Charmaz, 2013), inicialmente en una codificación abierta, a partir de la precodificación y los códigos in vivo, literales (Bonilla \& López, 2016) cómo que «llegar a viejo es ser un estorbo».

Buscando "crear categorías teóricas a partir de los datos y analizar las relaciones" (Charmaz, 1990, citado en De la Cuesta, 2006, p. 137), los elementos en común posibilitaron codificar con mayor integración. Andréu et al. (2009) sugieren “comenzar la codificación axial desde el principio" (p. 72), ya ahí se configuraban categorías cómo «dependencia», recurrentes.

Los datos indujeron su teoría (Massone, 2010). Una tabula rasa y un diseño flexible "según se va desarrollando la investigación (...) [incorporando] puntos de vista de los participantes, los cuales no se conocen ni comprenden al iniciar" (Salamanca \& Martín-Crespo, 2007).

Se depuró la información en torno a "categorías», "condiciones», «acciones e interacciones» y «consecuencias» apegadas al relato, "más allá de los estudios previos (...) en búsqueda de nuevas formas de entender los procesos sociales" (Salgado, 2007, p. 72). La discusión exhaustiva permitió no perder elementos prominentes ni imponer ninguna mirada a priori. Así, se trasciende lo específico ensamblando "una denominación común [en torno a] a un conjunto de datos que 
comparten una misma idea" (Soneira, 2006, p. 156). Empiezan a aparecer repeticiones, imposibles de abstraer ex ante que revelan inquietudes compartidas.

"La codificación selectiva, continúa la codificación axial en un nivel más alto" (Flick, 2004, p. 198) integrando todo en un solo paradigma. Se alcanzan a "descubrir patrones en los datos, así como las condiciones bajo las cuales se aplican" (Flick, 2004, pp. 198-199) construyendo finalmente una teoría acerca de los significados del DS durante la AM para los participantes.

\section{Construcción del modelo comprensivo resultante}

De los DDSS que los AAMM conocen, emerge la «injusticia social»: los AAMM no se sienten considerados y sobre sí, relatan sentirse "una persona que sabe que recibe el mínimo, que tiene y que puede trabajar y que no tiene dónde... ni el cómo trabajar". Otra entrevistada expone, "para los jóvenes, los adultos son una molestia (...) para la sociedad en general". Esta injusticia queda explícita cuando una participante señala que ser AM; "tiene mucho de negativo con respecto a la mayoría de las personas que tienen unas pensiones bajísimas, es lo principal (...) entonces les cuesta mucho vivir o sobrevivir".

EI DS de «dependencia» también conlleva un fuerte mensaje implícito y da cuenta de la relevancia que parece tener la autovalencia. Se relata, "puedo hacerme cargo, tengo para cubrir y no tengo que andar pidiendo (... ) yo puedo, yo puedo".

Ambas ideas se interrelacionan generando disonancia. La «afectividad» surge cómo respuesta y ayuda a comprender ansiedades y miedos, en un sistema que por un lado no da oportunidades, pero dónde no ser autovalente adquiere una connotación muy negativa. Se asoman impotencias, rabias e inseguridades: eventualmente devienen sentimientos de abandono: "el AM es muy discriminado, porque donde uno vaya ya sea hospitales, no le dan las atenciones eficaces (...) el AM y es un estorbo hasta para los médicos".

La vivencia de un «trato indigno» también comprende esa interacción entre «injusticia social» y de «dependencia». Uno señala, "yo lo viví con mi esposa fallecida en el 2018, pedía la ambulancia y la espera era de horas, con frío una persona de edad, delicada, esperando que la atiendan, para mí es duro". Se trata de expectativas no cumplidas y necesidades mínimas no cubiertas a nivel sociosanitario y se condice con la injusticia y el miedo a depender de otros.

Una participante se refiere a las residencias planteando que "los viejitos en la parte estatal, que son del gobierno, están todos meados, todos cagados (...) lloran porque tienen grietas debajo de los brazos (...) no hay agua... no se bañan (...) No es digno y ¡espero yo no llegar a eso!”. Se fragua aquí la idea de «marginación»: los AAMM son dejados a un lado e invisibilizados. 
Los participantes señalan que son vistos como «estorbo» o «cacho». Si hay frustración, soledad y abandono, en un sistema injusto dónde el AM no logra encontrar un rol, esto revela otro significado: la «autoexigencia» cómo necesidad de mantener la funcionalidad. Perderla, se asocia a gran malestar y hacer frente a una realidad extremadamente injusta.

Figura 1. Proceso de significación de los DDSS en los AAMM, Diagramación del modelo resultante.

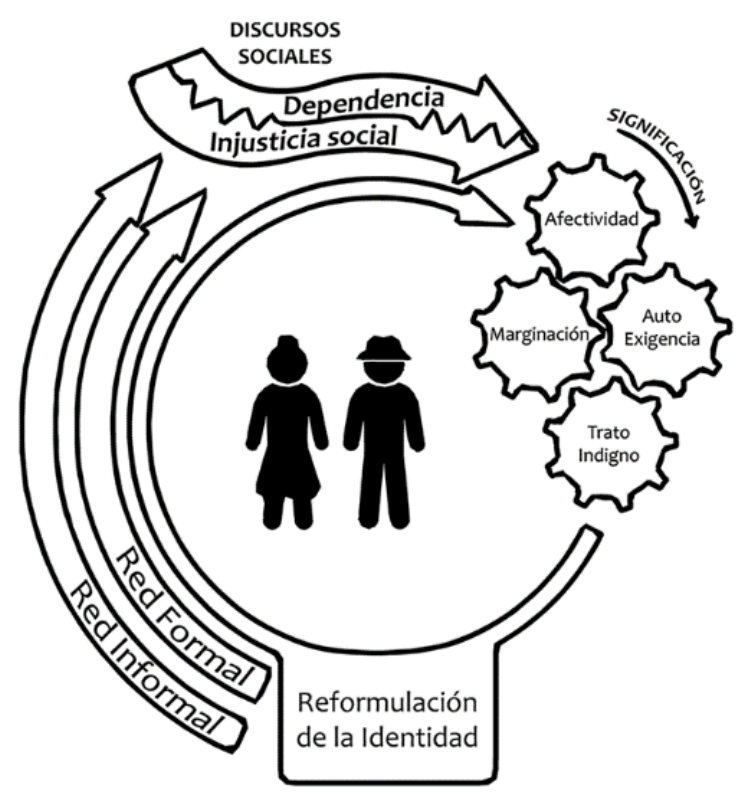

Fuente: Elaboración propia.

Todas las construcciones que los AAMM usan para dar sentido al DS interactúan en relación con la historia de vida del sujeto y su vinculación con el sistema de «redes informales». Se cierra aquí un ciclo afectando la «reformulación de la identidad». Una participante expone, "mi papá (...) trabajó y él fue el ejemplo de vida (...) [y yo] luché para salir adelante por mi hija, la eduqué. Mi hija también tiene el mismo ejemplo". Igualmente, la persona puede también recurrir a las «redes formales», señalan "nos hace sentir con un calor, con una donde el corazón salta más, ellos te saludan, te dan un abrazo (...) yo estoy feliz de haber compartido en los grupos del AM", "encontré el CIAM (... ) y la vida le cambia a uno (...) somos todos pares iguales".

\section{Conclusiones, discusión y reflexiones en torno a los hallazgos}

Hemos podido observar cómo, mediante su relato, los participantes dan entender que tanto la injusticia social cómo el miedo a la dependencia, se engranan en una afectividad asociada a la marginación, el trato indigno y la autoexigencia. La mayoría de los entrevistados tienen muy internalizada la creciente injusticia a medida que se envejece y la aparente importancia de mantener la autovalencia de forma casi 
indefinida. En la dinámica de reformulación identitaria, tales significados favorecen determinadas formas de interactuar.

Si esto redunda en cómo se va reformulando la identidad y en las formas de relacionarnos socialmente, necesariamente también toca el cómo, durante la $A M$, mediante redes, la persona logra o no hacerse parte activa del sistema social. Observamos un interés social evidente por propulsar lo que actualmente se denomina «envejecimiento activo», en muchos de los ejemplos que los mismos AAMM relatan. Pero pareciera que algo falta: a nivel de significados, nuestra sociedad no ha sido capaz de integrar al AM con roles nítidos y oportunidades genuinas de desarrollo.

\section{¿Cómo funcionan las redes de apoyo durante la adultez mayor?}

Las redes son críticas en la construcción de la identidad, en situaciones de aislamiento, enfermedad, crisis o abandono. Promueven y motivan la participación potenciando el funcionamiento, otorgando un sentido de pertenencia, consolidando amistades, entregando reconocimiento, sensación de bienestar y fortaleciendo la autoestima.

Ante el crecimiento de la población $\mathrm{AM}$, cabe reflexionar aquí sobre las políticas públicas pues también inciden en las redes y construcción de la identidad, especialmente desde el discurso que se promueve y su significado. A nivel de gobierno, del SENAMA y municipalmente existen diversos programas en ayuda del $\mathrm{AM}$, particularmente promotores del «envejecimiento activo», concepto que aporta a cambiar la percepción negativa de la vejez (Rojas, 2017).

Por otro lado, observamos que la entrega de recursos es insuficiente para que los AAMM sigan socialmente activos e independientes. Los AAMM son estigmatizados y marginados, existiendo inseguridad, injusticia social y económica: una constante lucha por integrarse. Incluso en sus familias son considerados a menudo personas menos productivas.

Las personas mayores tienen derecho a entregar trabajo y conocimiento y sorprende la sabiduría en su forma de vivir. Todo esto apunta a realizar intervenciones en beneficio del bienestar de los AAMM, buscando que logren adaptarse mejor (Erikson, 1985) ¿Pero cómo?

\section{¿Qué es lo que preocupa a la población adulta mayor?}

En aras de un país más justo, la sociedad debe tomar en consideración la riqueza que entrega la experiencia vivida con un sentido de plenitud integrativo. Es necesario cambiar, desde los más jóvenes, el DS dirigido a la vejez. Para estos últimos se observa como una desgracia (Arnold, Thumala \& Urqueza, 2007). 
Las políticas públicas aparentan orientarse al entretenimiento y la funcionalidad, más el relato de los participantes, repleto de alusiones a la marginación, está atravesado por ejemplos de los beneficios y los recursos. Entonces ¿qué es lo que realmente preocupa? ¿por qué y cómo, aparece este cuestionamiento y este descontento en lo social?

Los "criterios prejuicios acerca del valor social y productivo relativo a la edad" que describe lacub (2014, p. 188) hacen de esta etapa una muy distinta. El discurso de injusticia social es tal que llega a ser una de las preocupaciones que más mueve a los AAMM, junto a la dependencia, pues del miedo aparece una expectativa autoexigente irrealizable de no llegar a depender de nada ni nadie. Aquí, también la interrelación de los participantes es sus redes da mayor o menor fuerza a los discursos de injusticia social y dependencia antes señalados.

\section{¿Cómo trata la sociedad a los adultos mayores y que se espera de ellos?}

Ya Erikson (1985) señalaba que "los pacientes viejos parecen hacer un duelo (...) por la autonomía debilitada, la iniciativa abandonada, la intimidad faltante, la generatividad descuidada (...) la vivencia de una identidad demasiado limitadora" (p.80). Los participantes expresan ya no tener oportunidades de desarrollarse. Se «enfrentan» a la AM: su relato refleja temores, frustraciones, marginación y autoexigencia para integrado tratado dignamente.

Para Erikson (1985) “es la última ritualización incorporada al estilo de la vejez (...) [el] mantener algún orden y significado [lo que] puede defender una esperanza duradera de sabiduría" (p. 81). Pero, sin oportunidades, la sociedad subraya el valor de no depender. Esta cuestión deseable pero impracticable es precisamente la injusticia social que se devela en esta investigación, una que tiene un gran costo afectivo para los participantes. ¿Cuán justo socialmente es esperar de los AAMM algo casi imposible? ¿Cuáles son las oportunidades de desarrollo identitario para quienes evalúan que inexorablemente se vuelven dependientes? ¿Podría el hecho de fomentar el «envejecimiento activo» estar intensificando la autoexigencia?

Desde las redes se conjuga toda una historia vital que interactúa con la significación del DS. Para Becker (1992), cada quien interpreta, organiza su universo y atribuye significados propios, originando discursos dispares. Una participante exterioriza, "tengo una hija menor que me dice: mamá yo que haría si usted no, no fuera como está, tan autovalente como es usted". No se permite enfermar, necesita mantenerse activa. Igualmente, el sistema de redes motiva a «envejecer activamente», pero en parte también lo exige.

Durante la AM hay una necesidad de "contribuir al bien común de los entornos en los que las personas participan (...), reforzar y enriquecer las instituciones sociales, asegurar la continuidad entre generaciones o plantear mejoras sociales" (Villar, 
López \& Celdrán, 2013, p. 897). No es sólo evitar el deterioro ¿Hasta dónde entonces los DDSS ayudan a un proceso de envejecimiento coherente entre lo que se «dice» y lo que se «imparte»? A medida que se pierden roles ¿qué está sucediendo a fondo con la reformulación de la identidad?

\section{¿Qué rol y qué identidad termina por adoptar el adulto mayor?}

La identidad conlleva "cambios normativos inherentes al proceso de envejecimiento (... ) que pueden desafiar el sentido de sí" (lacub, 2014, p. 144). Pero ¿cuál es el rol de los AAMM? Desde la adultez media, para Erikson (1985) hay necesidad "de nuevos productos e ideas, incluido un (... ) desarrollo de la identidad" (p. 85), camino para el desafío final de su integración.

El discurso del «envejecimiento activo», sano, sin duda, e importante en lo sociosanitario, no sólo moviliza afectos, predispone a los AAMM a procesos autoabsorbentes a pesar de que se esperaría "un compromiso ampliado de cuidar de las personas, los productos y las ideas por los que uno ha aprendido a preocuparse" (Erikson, 1985, p. 85). Todo, en una sociedad que parece sobrevaluar lo novedoso con una mirada desechable de las cosas y de las relaciones.

Cómo "intereses y comportamientos generativos en la vejez tienen, además, relación con el bienestar” (Villar, López \& Celdrán, 2013, p. 903), la afectividad caracterizó el relato de los participantes. Sin un papel que desempeñar, sufren en el hoy lo que puede o no puede ser mañana. Viven en una sociedad dónde las puertas para ser parte integrante se les cierran.

Los participantes temen depender y sienten que la sociedad es injusta muy posiblemente debido a esta falta de roles. Sólo se centran en evitar lo inevitable mediante el «envejecimiento activo». Para ellos, se trata casi de una amenaza de indignidad y marginación. La autoexigencia, más que reformular la identidad, parece permitirles evitar hacerlo. A la base, entienden que en Chile no se admite la participación en el rol de AAMM como tales.

La dependencia es una de las mayores preocupaciones del AM en Chile (Herrera et al., 2017) pues la legislación no asegura un ingreso económico suficiente, ni derecho a servicios sociosanitarios adecuados, ni menos, a cumplir el sueño de una vejez digna. Así, estas tensiones se pudieron evidenciar en las marchas con en el descontento social de octubre 2019.

\section{Otras proyecciones y oportunidades a partir de esta investigación}

Se han perfilado algunos componentes clave del DS durante la AM, en una sociedad que parece no guardar un espacio para ellos. Mediante una investigación cuantitativa evaluando la representatividad o generando nuevos instrumentos para medir variables como dependencia o marginación podría profundizarse. Con ello se pueden 
implementar nuevas políticas públicas disminuyendo las discrepancias entre el DS, el «envejecimiento activo», y lo que los roles sociales actuales admiten cuando perpetúan fácticamente la marginación.

Chile enfrenta un envejecimiento acelerado, un desafío para los CIAM que deben otorgar oportunidades de integración y participación. De un total de sesenta AAMM en el centro «Lucía Salas Romo» sólo diez son hombres. Pero el CIAM es abierto para todos ¿cuáles podrían ser las razones? Incluso de este tipo de centros, Ortega destaca (2018) la:

Sobre representación femenina, la cual también debe ser leída bajo la reproducción de un paradigma patriarcal donde se constituyen determinados patrones de lo femenino y lo masculino (p. 230).

¿Cursaran entonces estereotipos de por medio? ¿Temas de género u otros prejuicios?

\section{Referencias}

Aho, K. (2018). Temporal experience in anxiety: embodiment, selfhood, and the collapse of meaning. Phenomenology and the Cognitive Sciences, 17(1), 1-12.

Andréu, J., García-Nieto, A., y Pérez, A. (2009). Componentes clave de la grounded theory. En Cuadernos Metodológicos 40: Evolución de la Teoría Fundamentada como Técnica de Análisis Cualitativo (pp. 53-79). Centro de Investigaciones Sociológicas.

Arnold, M., Thumala, D., Urquiza, A., \& Ojeda, A. (2007). La vejez desde la mirada de los jóvenes chilenos: estudio exploratorio. Última Década, 15(27), 75-91. http://dx.doi.org/10.4067/S0718-22362007000200005

Bandura, A. (1988). Self-efficacy conception of anxiety. Anxiety Research, 1(2), 77-98.

Benito, R. (2013). Adaptación y resistencia al orden social neoliberal en Chile: habitus y discursos sociales, el caso de la Región de Antofagasta. [Tesis doctoral inédita]. Universitat de Valencia.

Blumer, H. (1982). El interaccionismo simbólico, perspectiva y método. Hora.

Bordignon, N. (2005). El desarrollo psicosocial de Eric Erikson: el diagrama epigenético del adulto. Revista Lasallista de Investigación, 2(2), 50-63.

Charmaz, K. (2006). Constructing grounded theory: a practical guide through qualitative analysis. Sage Publications.

Chóliz, M., y Gómez, C. (2002). Emociones sociales: enamoramiento, celos, envidia y empatía. En Psicología de la Motivación y Emoción (pp. 395-418). McGraw Hill.

Colegio de Psicólogos de Chile. (1999). Código de ética profesional. Colegio de Psicólogos de Chile, Asociación Gremial. 
Delval, J. (1997). Hoy todos son constructivistas. Cuadernos de Pedagogía, 257, 78-84.

De la Cuesta, C. (2006). La teoría fundamentada como herramienta de análisis. Cultura de los cuidados, 20(2), 136-140.

Erikson, E. (1985). El ciclo vital completado. Paidós.

Flick, U. (2004). Introducción a la investigación cualitativa ( $3^{\circ}$ ed.). Morata.

Foucault, M. (2005). El orden del discurso. Fabula Tus Quets Editores.

Gergen, K. (1996). Realidades y relaciones: aproximación a la construcción social. Paidós Ibérica.

Gergen, K. (2007). Constructivismo social: aportes para el debate y la práctica. Uniandes.

Hernández, R., Fernández, C., y Baptista, P. (2014). Metodología de la investigación (6ta ed.). McGraw-Hill.

Herrera, M., Fernández, M., Rosell, J., y Rojas, M. (2017). Chile y sus mayores. Caja los Andes.

lacub, R. (2014). Identidad y envejecimiento: perspectivas de occidente. Paidós.

Iñiguez, L., y Antaki, C. (1994). El análisis del discurso en psicología social. Boletín de Psicología, 44(63), 57-75.

Izquierdo, Á. (2006). Psicología del desarrollo de la edad adulta Teorías y contextos. Revista complutense de educación, 16(2), 601-619.

Katayama, R. (2014). Introducción a la Investigación Cualitativa: Fundamentos, métodos, estrategias y técnicas. Universidad Inca Garcilaso de la Vega. Editorial UIGV.

Lazarus, R. (2000). Epistemología y metateoría. En Estrés y Emoción: Manejo e Implicaciones en Nuestra Salud (pp. 1-18). Desclée de Brouwer.

Martínez, M. (2006). La investigación cualitativa (síntesis conceptual). Revista de Investigación en Psicología, $\quad$ 9(1), 123-146. http://dx.doi.org/10.15381/rinvp.v9i1.4033

Massone, F. (2010). Experiencias del cuerpo en movimiento durante la vejez: actividad física recreativa y calidad de vida en adultos mayores. Universidad de Chile.

Mead, G. (2008). La filosofía del presente. Boletín Oficial del Estado.

Mead, M. (2006). Sexo y temperamento en tres sociedades primitivas. Paidós.

Moñivas, A. (1998). Representaciones de la vejez (modelos de disminución y de crecimiento). Anales de Psicología, 14(1), 13-25. http://dx.doi.org/10.6018/analesps 
Neugarten, B. (1999). Los significados cambiantes de la edad. En los Significados de la Edad. (pp. 75-83). Herder.

Noreña, A., Alcaraz, N., Rojas, J., y Rebolledo, D. (2012). Aplicabilidad de los criterios de rigor y éticos en la investigación cualitativa. Aquichan, 12(3), 263-274.

Organización Mundial de la Salud. (2020). Infecciones por coronavirus. https://www.who.int/topics/coronavirus_infections/es/

Ortega, D. (2018). Envejecimiento y trato hacia las personas mayores en Chile: una ruta de la desigualdad persistente. Sophia Austral, 22, 223-246.

Papalia, D., Feldman, R., y Martorell, G. (2012). Desarrollo Humano (12ava ed.). McGraw Hill.

Patillo, G. (2018). Estimaciones y proyecciones de la población de Chile 1992-2050, síntesis de resultados. Instituto Nacional de Estadísticas.

Pernalete, D., Peña, K., y Pérez, M. (2016). Decisiones para el uso de la teoría fundamenta en la tesis doctoral. Anuario del Doctorado en Educación: Pensar en la Educación, 8, 61-72.

Rojas, M. (2017). Biogerontología (No. 6, Psicogerontología). Universidad UNIACC.

Salamanca, B., y Martín-Crespo, C. (2007). El diseño en la investigación cualitativa. Nure Investigación, 26.

Salgado, C. (2007). Investigación cualitativa: diseños, evaluación del rigor metodológico y retos. Liberabit, 13(3), 71-78. Discursos Sociales

Sandoval, J. (1997). Producción discursiva y problemas sociales: el ejemplo de la construcción social del problema del consumo de pasta base. Última Década, 7.

Sandoval, J. (2009). Representaciones de la ciudadanía en los discursos del «saber experto»: la individualización de la desigualdad. Psicoperspectivas, Individuo y Sociedad, 8(2), 35-56.

Soneira, A. (2006). La «teoría fundamentada en los datos» (grounded theory) de Glaser y Strauss. En Estrategias de Investigación Cualitativa (pp. 153-173). Gedisa.

Thumala, D. (2011). Formas de afrontamiento a las pérdidas asociadas al envejecer.

Valles, M. (2009). Cuadernos metodológicos 32: entrevistas cualitativas. Centro de Investigaciones Sociológicas.

Young, R., y Collin, A. (2004). Introduction: Constructivism and social constructionism in the career field. Journal of Vocational Behavior, 64(3), 373-388.

Madariaga, A., y Núñez, V. (2008). Aspectos estadísticos del Cuestionario de Calidad Revista Pensamiento Académico de la Universidad UNIACC 
de Vida relacionada con salud. Estadística Española, 50(167)

Charmaz, K. (2013) La teoría fundamentada en el siglo XXI: Aplicaciones para promover estudios sobre la justicia social, pp. 270-325 En: N. K. Denzin; Y S. Lincoln (comps.) Estrategias de investigación cualitativa: Vol. III. Gedisa.

Bonilla, M., y López, A.. (2016). Ejemplificación del proceso metodológico de la teoría fundamentada. Cinta de Moebio, (57), 305-315.

San Martin, D. (2014) Teoría fundamentada y ATLAS. ti: recursos metodológicos para la investigación educativa. Revista electrónica de investigación educativa, 16(1): 104-122.

Villar, F., López, O. y Celdrán, M. (2013). La generatividad en la vejez y su relación con el bienestar: ¿quién más contribuye es quien más se beneficia? Anales de psicología, 29(3), 897-906. 


\section{Jesús Aparicio de Soto}

Psicólogo (colegiado №6902), Ingeniero Civil (colegiado №27971-4) y SCRUM Master Certificado. Cursos en Gestión Remota (PMI), Factores Culturales \& UX (Univ. Tsinghua), Representación del Razonamiento (Inst. Guwahati), Diseño Instruccional AGILE (LinkedIn), PNL (Alison). Certificado Google en Cloud, Desarrollo Móvil y Negocios Digitales. Participó en I+D Innova CORFO (economizador modular), extractor esencias vinícolas CONICYT, desarrollo web UAI, fue ayudante del profesor (UML y POO) e integró equipo Atención Psicoeducativa UNIACC. Líder, gestor y arquitecto de experiencias humano-sistema cognitivamente ergonómicas. Director Centro Extiende.cl psicoterapia remota. Experiencia psicoterapéutica, psicoeducativa y cómo facilitador de grupos, foco atención remota y Tls.

Email:correo.jesus.aparicio@gmail.com

\section{Susana Cornejo Farías}

Licenciada de psicología y grado académico de Psicóloga, Universidad UNIACC, Chile. Diplomado en Mediación Familiar, Educación Central Limitada (acreditado). Curso de perfeccionamiento Manejo de la Depresión en Personas Mayores, IPLACEX. Curso Herramientas de Apoyo a la Función del Investigador de la Denuncia y de la Investigación de la Violencia en el Trabajo, IPLACEX. Curso Bioética Aplicada a la Salud Pública, realizado en IPLACEX. Curso Habilidades Psicosociales Básicas en Contexto de COVID-19 para el Personal de Salud, IPLACEX. Curso Formación en Primera Ayuda Psicológica (PAP) en Contexto de COVID- 19, IPLACEX. Curso Prevención del Suicidio para Equipos de Salud de Hospitales, IPLACEX. Curso Psicología del Aprendizaje, Universidad de Palermo (Buenos aires, Argentina).

Email: susanacornejofarias@gmail.com

\section{Francisca Lain Soteras}

Administradora formada en Instituto Profesional INACAP, con experiencia en gestión de proyectos y licitaciones nacionales e internacionales. Participó en el rubro turístico privado y en el programa de reconstrucción (terremoto 2010) del Ministerio de Vivienda y Urbanismo. Posteriormente, obtiene el grado académico de Psicóloga en la Universidad UNIACC. Actualmente, se desempeña como psicóloga clínica y como Coordinadora del Equipo de Servicios Especializados del Programa Nacional de Apoyos y Cuidados en Linares, Chile.

Email: panchalain@yahoo.com

\section{Paula Mallegas Roblin}

Psicóloga Clínica Universidad de Artes, Ciencias y Comunicación UNIACC, Santiago de Chile. Consejera Orientadora Familiar del Centro Nacional de la Familia (CENFA). Co-fundadora y directora del Centro de Orientación Mutinelli (CORFAM), desde el 2015 - 2020. Experiencia de más de diez años en el ámbito de la terapia familiar, de parejas y familias en el sistema público y privado. Actualmente cursando diplomado en Estrategias de Evaluación e Intervención en la Infancia, modelo AMAR del Ph. Felipe Lecannelier, por otra parte, realizando el curso en Evaluación del Funcionamiento Reflexivo (FR) en la Parent Development Interview (PDI), acreditado por el Instituto Universitario de Salud Mental Fundación Vidal y Barraquer, Universidad Ramón Llull, Barcelona.

Email: paulamallegas@yahoo.es 